\title{
Los Cuidados Paliativos. Un nuevo reto para la Medicina Interna
}

\author{
Palliative Care. A new challenge for Internal Medicine
}

\section{Miguel Ángel Núñez Viejo}

Unidad de Cuidados Paliativos. Complejo Hospitalario Universitario de Ourense

Palabras clave: Cuidados Paliativos. Cuidados continuos. Medicina Interna.

De un lado, el envejecimiento progresivo de la población y el consiguiente aumento exponencial de las patologías crónicas, la mejora en los tratamientos y el consecuente aumento en la sobrevida, la prevalencia cada vez mayor del cáncer y otra serie de factores sociosanitarios ${ }^{1}$; y del otro, la insuficiente adaptación de los profesionales de la salud al manejo del paciente crónico en situación de terminalidad, que con frecuencia conlleva una atención médica en muchos casos desproporcionada y con consumo de gran cantidad de recursos (lo que se conoce como encarnizamiento terapéutico u obstinación terapéutica), hacen necesario y acuciante el desarrollo e implementación de programas de cuidados paliativos que garanticen una adecuada atención de los pacientes $^{1-3}$.

Al contrario de lo que puede parecer, la atención a la cronicidad avanzada y la enfermedad terminal sigue siendo un reto del Sistema Nacional de Salud, cuyo objetivo debe ser simplificar los métodos de identificación del paciente crónico con edad avanzada y las necesidades de cuidados paliativos. En este sentido, los distintos estudios de prevalencia de que disponemos hablan de que hasta el 1,4-26\% de los pacientes que están en domicilio podrían beneficiarse de cuidados paliativos, y este porcentaje se eleva al $66-70 \%$ cuando tenemos en cuenta las instituciones sociosanitarias ${ }^{3,4}$.

Así, tanto en el ámbito de la Atención Primaria como en el medio hospitalario, pocos son los pacientes (se estima que en torno a un 15-20\%, según las series) que entran en programas de cuidados paliativos ${ }^{5}$, casi siempre en unidades específicas y enfermos en su mayoría con patología oncológica. Aplicando de manera estricta los criterios de enfermedad terminal definidos para cada patología, las estadísticas no son mucho mejores si nos fijamos en los servicios de Medicina Interna, donde menos de un tercio de los pacientes que tienen enfermedades crónicas no oncológicas (insuficiencia renal, hepatopatía, enfermedad cerebrovascular, insuficiencia cardiaca, EPOC, VIH/SIDA, etc.) en fase avanzada y situación clínica de terminalidad son reconocidos como tales por el médico que les atiende ${ }^{6}$, lo que hace más difícil aún la limitación de los esfuerzos diagnósticos y terapéuticos y conlleva que estos a menudo sean, como ya se ha mencionado, desproporcionados.

Como resumen, podría afirmarse que en el conjunto del Sistema Nacional de Salud sólo el 30-35\% de los pacientes que fallecen anualmente en España por una enfermedad terminal reciben cuidados paliativos específicos, a través de los equipos de Atención Primaria, las unidades de atención domiciliaria 0 en las unidades hospitalarias de agudos, media y larga estancia.

Pero éste es un hecho cambiante en los últimos años, y un factor clave del proceso ha sido el cambio radical en la demanda social, que ha identificado la promoción de los cuidados paliativos como un elemento cualitativo esencial del sistema de salud, de forma que la puesta en marcha de programas de Cuidados Paliativos en los planes de Salud es un indicador del sistema sanitario para el bienestar de sus ciudadanos ${ }^{7,8}$. Otro punto clave de este cambio es la mayor atención y formación de la Atención Primaria en el manejo de este tipo de enfermos. El objetivo principal de Ios Cuidados Paliativos debe ser por tanto la promoción del confort y de la calidad de vida del paciente y de la familia, basada en el control de síntomas, el soporte emocional y la comunicación.

Para dar respuesta a esta demanda, en 1992 se formó la Sociedad Española de Cuidados Paliativos (SECPAL), como institución reguladora y unificadora de criterios y estándares mínimos de atención. Poco después, en 1999 y a raíz de la Recomendación 1418 del Consejo Europeo, el Pleno del Senado aprobó una moción por la que se instaba al Gobierno a elaborar un Plan Nacional de Cuidados Paliativos en el marco del Consejo Interterritorial del Sistema Nacional de Salud. En cumplimiento de este mandato se elaboró en diciembre de 2000 el documento denominado "Plan de Cuidados Paliativos, bases para su desarrollo". Otros hitos importantes fueron el desarrollo de la Estrategia Nacional de Cuidados Paliativos de 2007, y más específicamente en nuestra comunidad, el Plan Galego de Coidados Paliativos ${ }^{2}$ de 2005. Igualmente cabe destacar la labor fundamental de las Unidades de Hospitalización a Domicilio, que hasta entonces habían llevado el peso de la atención de este tipo de enfermos.

Con todo ello se definió entonces el concepto de Enfermedad Avanzada y Compleja (EAC) como aquella que por su historia natural y/o complicaciones diagnóstico-terapéuticas genera en el paciente y la familia necesidades de difícil solución y gran impacto físico, psicológico, social o espiritual, y que con frecuencia acostumbra a desembocar en la etapa de 
enfermedad-enfermo terminal. Sin embargo, tratamientos 0 cuidados paliativos no es equivalente a paciente terminal 0 etapa terminal. Entonces, ¿qué se entiende por enfermos terminales?. Partiendo del hecho que no es un término apropiado, pues su uso conlleva connotaciones despectivas, si tuviésemos que hablar de terminalidad, habría que distinguir entre la terminalidad terapéutica, cuando el paciente ya no se beneficia de ningún tratamiento activo bien porque esté demostrada su ineficacia o bien porque esté contraindicado -nos centraremos por tanto en cuidados y manejo de síntomas-, y la terminalidad clínica o biológica (un paciente con insuficiencia orgánica y expectativa de vida corta, o bien en situación de preagonía o agonía).

Los Cuidados Paliativos abarcan por tanto la asistencia total, activa y continua de los pacientes y sus familias. Estos cuidados deben ser aplicados por un equipo multiprofesional cuando la expectativa médica no es la curación, y su meta fundamental es dar calidad de vida al paciente y su familia, sin intentar prolongar la supervivencia3,9-11.

En su desarrollo debería jugar un papel muy importante la Medicina Interna, pues como ya se ha mencionado, el patrón demográfico actual de morbilidad y de progreso de la medicina hace esperar que en los próximos años aumente el número de personas con cáncer avanzado y con enfermedades crónicas y degenerativas. Además, cada día son más los médicos internistas que por diferentes circunstancias desarrollan su labor asistencial en unidades de cuidados paliativos. Por todo lo mencionado, es muy importante tener conocimientos en este ámbito y fundamental la formación, cada vez más amplia, en esta disciplina, para saber adecuar y adaptar nuestras acciones terapéuticas al grado de evolución de la enfermedad de nuestros pacientes.

Implantar un proyecto como éste en el ámbito de la Medicina Interna ${ }^{5,10,12}$ requiere combinar dos tipos de estrategias $^{13,14}$. En primer lugar, la formación, cuanto menos básica, en Cuidados Paliativos de todos los profesionales sanitarios $y$, en segundo lugar, el desarrollo de programas específicos más amplios de Cuidados Paliativos que otorguen a determinados profesionales la posibilidad de intervención en todos los niveles asistenciales y la cualificación necesaria para la atención de los casos más complejos. Para ello lo ideal es optimizar los recursos disponibles, de forma que creando un grupo de trabajo al respecto dentro de la sociedad se contaría además con un criterio unánime y una misma línea de actuación. Sin embargo, no debemos olvidar que debido a las características de los enfermos existen serias limitaciones para la práctica de la investigación, lo que impide el desarrollo de una base firme de conocimientos y habilidades metodológicas en esta disciplina, inquietudes básicas de todo buen internista

Es fundamental que la Sociedad Española de Medicina Interna (SEMI) tenga representación y se haga escuchar en aquellas instituciones que se desenvuelvan en el ámbito de los Cuidados Paliativos, y para ello debe promover desde los hospitales y otros estamentos programas de atención inte- gral de los pacientes con enfermedades avanzadas dentro y fuera del propio hospital 6,13,14. Las razones definitivas por tanto, podrían quedar resumidas de la siguiente forma:

- Se hace necesaria, teniendo en cuenta el tipo de pacientes que tratamos, la formación de calidad del personal sanitario en general y de los facultativos de Medicina Interna en particular en el área de los Cuidados Paliativos.

- Esta justificación tiene su origen en la demanda creciente de cuidados paliativos por parte de la Sociedad, que se ha convertido en una de las necesidades de mayor trascendencia social. Para ello es obligada la elaboración de un currículo profesional en cuidados paliativos para todo el personal sanitario.

- La Medicina Interna aportaría al resto de especialidades implicadas la capacidad de asesoramiento y puesta al día en los avances en cuidados paliativos a través de un modelo de intervención interdisciplinaria que orientara el trabajo de cara al paciente y su familia, para así dar a conocer los problemas clínicos y las situaciones relevantes del paciente en situación avanzada y terminal con intención de mejorar las habilidades diagnósticas y terapéuticas.

En conclusión, el ámbito de la Medicina Interna es el óptimo para este tipo de enfermos, y fundamental el papel del internista $7,12,14,15$, porque somos una especialidad capaz de proporcionar una orientación bajo un enfoque fisiopatológico, diagnóstico y terapéutico global.

Como solución a esta situación surgió hace escasamente dos años, a raíz de la inquietud nacida en varios compañeros internistas, la idea de crear un Grupo de Trabajo sobre "Cuidados Paliativos y Tratamiento del Dolor", con objeto de, bajo los auspicios de la SEMI, coordinar de una forma más adecuada la asistencia, docencia e investigación en relación con este tema. La propuesta fue finalmente presentada por profesionales pertenecientes bien a Unidades de Hospitalización de Cuidados Paliativos, Equipos de Soporte de Cuidados Paliativos, Unidades de Hospitalización de Crónicos y Atención Continuada y Unidades de Hospitalización a Domicilio adscritos a diversos hospitales españoles, mostrando este hecho el carácter multidisciplinar que debe caracterizarnos.

La respuesta de la SEMI fue inicialmente aconsejar nuestra adhesión a alguno de los grupos de trabajo ya existentes (Hospitalización a Domicilio y Telemedicina, Paciente Pluripatológico y de Edad Avanzada...), a fin de no solapar ámbitos de atención, pero es nuestro deseo que finalmente el grupo tenga un carácter independiente y sea aceptado por la junta directiva en próximas reuniones, para así poder emprender con paso firme esta nueva andadura y establecer líneas de trabajo (monografías, boletines informativos, encuestas de opinión, página web) y futuros protocolos, así como proyectos de investigación multicéntricos. Esto justificó una primera reunión en noviembre de 2015 en Sevilla, durante la celebración del XXXVI Congreso Nacional de la Sociedad Española de Medicina Interna, con la misión de 
elaborar unas bases de trabajo común. Somos conscientes de que como paso previo, es prioritario conocer los recursos con los que cuenta cada comunidad para el cuidado de los pacientes paliativos, pues existe una gran variabilidad de asistencia, no sólo en las diversas autonomías, sino también dentro de las propias ciudades o regiones. Pero igualmente primordial es sensibilizar a todos los médicos internistas de la necesidad de nuestros pacientes de tener una atención de calidad basada en Cuidados Paliativos en los estadios finales de enfermedades crónicas y avanzadas, así como publicitar y ofrecer cursos de formación al respecto, dirigidos fundamentalmente a residentes y abiertos a otros profesionales de la Medicina Interna. Es por ello que me gustaría acabar recordando que este grupo de trabajo está abierto a todos aquellos internistas que deseen participar en él, con ánimo de aportar su valiosa contribución.

\section{Bibliografía}

1. Camps C. ¿Hace falta una nueva concepción de los cuidados paliativos en oncología? Contrarréplica. Rev Oncol. 2003;5:305-7.

2. Guía del Plan Galego de Coidados Paliativos. Santiago de Compostela. Ed. Consellería de Sanidade. Servizo Galego de Saúde, 2007.

3. Sanz Ortiz J, Gómez Batiste X, Gómez Sancho M, Núñez Olarte J.M. "Cuidados Paliativos: Recomendaciones de la Sociedad Española de Cuidados Paliativos". Madrid. Ed.: Ministerio de Sanidad y Consumo, 1993.

4. Murtagh FE, Bausewein C, Verne J, Groeneveld El, Kaloki YE, Higginson IJ. How many people need palliative care? A study developing and comparing methods for population-based estimates. Palliat Med. 2014;28(1):49-58.

5. Navarro Sanz R. ¿Es la atención sanitaria a las personas mayores con enfermedad avanzada una alternativa asistencial de la Medicina Interna? An Med Interna (Madrid) 2004;21:315-16.

6. Quill TE, Abernethy AP. Generalist plus specialist palliative care-creating a more sustainable model. N Engl J Med. 2013;368(13):1173-5.

7. Gómez J. La Medicina Interna: situación actual y nuevos horizontes. An Med Interna (Madrid) 2004;21:301-5.

8. Schenker Y, Arnold R. The Next Era of Palliative Care. JAMA. 2015;314(15):1565-6.

9. Gott M, Gardiner C, Ryan T, Parker C, Noble B, Ingleton C. Prevalence and predictors of transition to a palliative care approach among hospital inpatients in England. $J$ Palliat Care. 2013;29(3):147-53.

10. Herrero F. La Medicina Interna actual. Una visión interesada. Rev Clin Esp. 2003;203:242-3.

11. Kelley AS, Morrison RS. Palliative Care for the Seriously III. N Engl J Med. 2015;373(8):747-55

12. Campo A. Estudio socioprofesional de la Medicina Interna en España: Prospectiva 2010. Ed. Sociedad Española de Medicina Interna (SEMI) 2005. P. 40, 44, 57, 61-5.

13. Sancho-Zamora MA. Medicina interna y cuidados paliativos. Rev Clin Esp. 2011;211(3):164-5.

14. Solano López D. El internista en los Cuidados Paliativos. Consultado en: http:// www. paliativossinfronteras.com/upload/publica/libros/cuidados-pal-labor-todos/06-EL-INTERNISTA-EN-LOS-CUIDADOS-PALIATIVOS-SOLANO.pdf

15. Losa JE, Zapatero A, Barba R, Marco J, Plaza S, Canora J; por el Grupo de Trabajo de Gestión Clínica de la Sociedad Española de Medicina Interna (SEMI). Encuesta de la SEMI sobre la Oferta Asistencial de los Internistas en el Sistema Público de Salud. Rev Clin Esp. 2011;211(5):223-32. 